\title{
ON $\mathbb{R}^{d}$-VALUED PEACOCKS
}

\author{
Francis Hirsch ${ }^{1}$ And Bernard RoynetTe ${ }^{2}$
}

\begin{abstract}
In this paper, we consider $\mathbb{R}^{d}$-valued integrable processes which are increasing in the convex order, i.e. $\mathbb{R}^{d}$-valued peacocks in our terminology. After the presentation of some examples, we show that an $\mathbb{R}^{d}$-valued process is a peacock if and only if it has the same one-dimensional marginals as an $\mathbb{R}^{d}$-valued martingale. This extends former results, obtained notably by Strassen [Ann. Math. Stat. 36 (1965) 423-439], Doob [J. Funct. Anal. 2 (1968) 207-225] and Kellerer [Math. Ann. 198 (1972) 99-122].
\end{abstract}

Mathematics Subject Classification. 60E15, 60G44, 60G15, 60G48.

Received July 26, 2011. Revised April 17, 2012.

\section{INTRODUCTION}

\subsection{Terminology}

First we fix the terminology. In the sequel, $d$ denotes a fixed integer and $\mathbb{R}^{d}$ is equipped with a norm which is denoted by $|\cdot|$.

We say that two $\mathbb{R}^{d}$-valued processes: $\left(X_{t}, t \geq 0\right)$ and $\left(Y_{t}, t \geq 0\right)$ are associated, if they have the same one-dimensional marginals, i.e. if:

$$
\forall t \geq 0, \quad X_{t} \stackrel{(\text { law })}{=} Y_{t} .
$$

A process which is associated with a martingale is called a 1-martingale.

An $\mathbb{R}^{d}$-valued process $\left(X_{t}, t \geq 0\right)$ will be called a peacock if:

(i) it is integrable, that is:

$$
\forall t \geq 0, \quad \mathbb{E}\left[\left|X_{t}\right|\right]<\infty ;
$$

(ii) it increases in the convex order, meaning that, for every convex function $\psi: \mathbb{R}^{d} \longrightarrow \mathbb{R}$, the map:

$$
t \geq 0 \longrightarrow \mathbb{E}\left[\psi\left(X_{t}\right)\right] \in(-\infty,+\infty]
$$

is increasing.

\footnotetext{
Keywords and phrases. Convex order, martingale, 1-martingale, peacock.

1 Laboratoire d'Analyse et Probabilités, Université d'Évry - Val d'Essonne, Boulevard F. Mitterrand, 91025 Évry Cedex, France. francis.hirsch@univ-evry.fr

2 Institut Elie Cartan, Université Henri Poincaré, B.P. 239, 54506 Vandœuvre-lès-Nancy Cedex, France.

bernard.roynette@iecn.u-nancy.fr
} 
This terminology was introduced in [5]. We refer the reader to this monograph for an explanation of the origin of the term: "peacock", as well as for a comprehensive study of this notion in the case $d=1$.

Actually, it may be noted that, in the definition of a peacock, only the family $\left(\mu_{t}, t \geq 0\right)$ of its one-dimensional marginals is involved. This makes it natural, in the following, to also call a peacock, a family $\left(\mu_{t}, t \geq 0\right)$ of probability measures on $\mathbb{R}^{d}$ such that:

$$
\forall t \geq 0, \quad \int|x| \mu_{t}(\mathrm{~d} x)<\infty
$$

(ii) for every convex function $\psi: \mathbb{R}^{d} \longrightarrow \mathbb{R}$, the map:

$$
t \geq 0 \longrightarrow \int \psi(x) \mu_{t}(\mathrm{~d} x) \in(-\infty,+\infty]
$$

is increasing.

Likewise, a family $\left(\mu_{t}, t \geq 0\right)$ of probability measures on $\mathbb{R}^{d}$ and an $\mathbb{R}^{d}$-valued process $\left(Y_{t}, t \geq 0\right)$ will be said to be associated if, for every $t \geq 0$, the law of $Y_{t}$ is $\mu_{t}$, i.e. if $\left(\mu_{t}, t \geq 0\right)$ is the family of the one-dimensional marginals of $\left(Y_{t}, t \geq 0\right)$.

Obviously, the above notions also are meaningful if one considers processes and families of measures indexed by a subset of $\mathbb{R}_{+}$(for example $\mathbb{N}$ ) instead of $\mathbb{R}_{+}$.

It is an easy consequence of Jensen's inequality that an $\mathbb{R}^{d}$-valued process which is a 1-martingale, is a peacock. So, a natural question is whether the converse holds.

\subsection{Case $d=1$}

A remarkable result due to Kellerer [6] states that, actually, any $\mathbb{R}$-valued process which is a peacock, is a 1 -martingale. More precisely, Kellerer's result states that any $\mathbb{R}$-valued peacock admits an associated martingale which is Markovian.

Two more recent results now complete Kellerer's theorem.

(i) Lowther [7] states that if $\left(\mu_{t}, t \geq 0\right)$ is an $\mathbb{R}$-valued peacock such that the map: $t \longrightarrow \mu_{t}$ is weakly continuous (i.e. for any $\mathbb{R}$-valued, bounded and continuous function $f$ on $\mathbb{R}$, the map: $t \longrightarrow \int f(x) \mu_{t}(\mathrm{~d} x)$ is continuous), then $\left(\mu_{t}, t \geq 0\right)$ is associated with a strongly Markovian martingale which moreover is "almost-continuous" (see [7] for the definition);

(ii) in a previous paper [4], we presented a new proof of the above mentioned theorem of Kellerer. Our method, which is inspired from the "Fokker-Planck Equation Method" ([5], Sect. 6.2, p. 229), then appears as a new application of M. Pierre's uniqueness theorem for a Fokker-Planck equation ([5], Thm. 6.1, p. 223). Thus, we show that a martingale which is associated to an $\mathbb{R}$-valued peacock, may be obtained as a limit of solutions of stochastic differential equations. However, we do not obtain that such a martingale is Markovian.

\subsection{Case $d \geq 1$}

Concerning the case $\mathbb{R}^{d}$ with $d \geq 1$, and even much more general spaces, we would like to mention the following three important papers.

(i) in [1], Cartier et al. study the case of two probability measures $\left(\mu_{1}, \mu_{2}\right)$ on a metrizable convex compact $K$ of a locally convex space. They prove, using the Hahn-Banach theorem, that, if $\left(\mu_{1}, \mu_{2}\right)$ is a $K$-valued peacock (indexed by $\{1,2\}$ ), then there exists a Markovian kernel $P$ on $K$ such that: $\theta\left(\mathrm{d} x_{1}, \mathrm{~d} x_{2}\right):=\mu_{1}\left(\mathrm{~d} x_{1}\right) P\left(x_{1}, \mathrm{~d} x_{2}\right)$ is the law of a $K$-valued martingale $\left(Y_{1}, Y_{2}\right)$ associated to $\left(\mu_{1}, \mu_{2}\right) ;$

(ii) in [8], Strassen extends the Cartier-Fell-Meyer result to $\mathbb{R}^{d}$-valued peacocks without making the assumption of compact support. Then he proves that, if $\left(\mu_{n}, n \geq 0\right)$ is an $\mathbb{R}^{d}$-valued peacock (indexed by $\mathbb{N}$ ), there exists an associated martingale which is obtained as a Markov chain; 
(iii) in [3], Doob studies, in a very general extended framework, peacocks indexed by $\mathbb{R}_{+}$and taking their values in a fixed compact set. In particular, he proves that they admit associated martingales. Note that in [3], the Markovian character of the associated martingales is not considered.

\subsection{Organization}

The remainder of this paper is organised as follows:

- in Section 2, we present some basic facts concerning the $\mathbb{R}^{d}$-valued peacocks and we describe some examples, thus extending results of [5];

- in Section 3, starting from Strassen's theorem, we prove that a family $\left(\mu_{t}, t \geq 0\right)$ of probability measures on $\mathbb{R}^{d}$, is associated to a right-continuous martingale, if and only if, $\left(\mu_{t}, t \geq 0\right)$ is a peacock such that the map: $t \longrightarrow \mu_{t}$ is weakly right-continuous on $\mathbb{R}_{+}$;

- in Section 4, by approximation from the previous result, we extend this result to the case of general $\mathbb{R}^{d}$-valued peacocks.

\section{Generalities, examples}

\subsection{Notation}

In the sequel, $d$ denotes a fixed integer, $\mathbb{R}^{d}$ is equipped with a norm which is denoted by $|\cdot|$, and we adopt the terminology of Section 1.1.

We also denote by $\mathcal{M}$ the set of probability measures on $\mathbb{R}^{d}$, equipped with the topology of weak convergence (with respect to the space $C_{b}\left(\mathbb{R}^{d}\right)$ of $\mathbb{R}$-valued, bounded, continuous functions on $\mathbb{R}^{d}$ ). We denote by $\mathcal{M}_{f}$ the subset of $\mathcal{M}$ consisting of measures $\mu \in \mathcal{M}$ such that $\int|x| \mu(\mathrm{d} x)<\infty$. $\mathcal{M}_{f}$ is also equipped with the topology of weak convergence.

$C_{c}\left(\mathbb{R}^{d}\right)$ denotes the space of $\mathbb{R}$-valued continuous functions on $\mathbb{R}^{d}$ with compact support, and $C_{c}^{+}\left(\mathbb{R}^{d}\right)$ is the subspace consisting of all the nonnegative functions in $C_{c}\left(\mathbb{R}^{d}\right)$.

\subsection{Basic facts}

Proposition 2.1. Let $\left(X_{t}, t \geq 0\right)$ be an $\mathbb{R}^{d}$-valued integrable process. Then $\left(X_{t}, t \geq 0\right)$ is a peacock if (and only if) the map: $t \longrightarrow \mathbb{E}\left[\psi\left(X_{t}\right)\right]$ is increasing, for every function $\psi: \mathbb{R}^{d} \longrightarrow \mathbb{R}$ which is convex, of $C^{\infty}$ class and such that the derivative $\psi^{\prime}$ is bounded on $\mathbb{R}^{d}$.

Proof. Let $\psi: \mathbb{R}^{d} \longrightarrow \mathbb{R}$ be a convex function. For every $a \in \mathbb{R}^{d}$, there exists an affine function $h_{a}$ such that:

$$
\forall x \in \mathbb{R}^{d}, \quad \psi(x) \geq h_{a}(x) \quad \text { and } \quad \psi(a)=h_{a}(a) .
$$

Let $\left\{a_{n} ; n \geq 1\right\}$ be a countable dense subset of $\mathbb{R}^{d}$. We set:

$$
\forall n \geq 1, \quad \psi_{n}(x)=\sup _{1 \leq j \leq n} h_{a_{j}}(x) .
$$

Then:

$$
\forall x \in \mathbb{R}^{d}, \quad \lim _{n \uparrow \infty} \uparrow \psi_{n}(x)=\psi(x) .
$$

The functions $\psi_{n}$ are convex and Lipschitz continuous.

Let $\phi$ be a nonnegative function, of $C^{\infty}$ class, with compact support and such that $\int \phi(x) \mathrm{d} x=1$. We set, for $n, p \geq 1$,

$$
\forall x \in \mathbb{R}^{d}, \quad \psi_{n, p}(x)=\int \psi_{n}\left(x-\frac{1}{p} y\right) \phi(y) \mathrm{d} y .
$$

Clearly, $\psi_{n, p}$ is convex, of $C^{\infty}$ class and Lipschitz continuous. Consequently, its derivative is bounded on $\mathbb{R}^{d}$. Moreover, $\lim _{p \rightarrow \infty} \psi_{n, p}=\psi_{n}$ uniformly on $\mathbb{R}^{d}$.

The desired result now follows directly.

The next result will be useful in the sequel. 
Proposition 2.2. Let $\left(X_{t}, t \geq 0\right)$ be an $\mathbb{R}^{d}$-valued peacock. Then:

1. the map: $t \longrightarrow \mathbb{E}\left[X_{t}\right]$ is constant;

2. the map: $t \longrightarrow \mathbb{E}\left[\left|X_{t}\right|\right]$ is increasing, and therefore, for every $T \geq 0$,

$$
\sup _{0 \leq t \leq T} \mathbb{E}\left[\left|X_{t}\right|\right]=\mathbb{E}\left[\left|X_{T}\right|\right]<\infty ;
$$

3. for every $T \geq 0$, the random variables $\left(X_{t} ; 0 \leq t \leq T\right)$ are uniformly integrable.

Proof. Properties 1 and 2 are obvious.

If $c \geq 0$,

$$
|x| 1_{\{|x| \geq c\}} \leq(2|x|-c)^{+} .
$$

As the function $x \longrightarrow(2|x|-c)^{+}$is convex,

$$
\sup _{t \in[0, T]} \mathbb{E}\left[\left|X_{t}\right| 1_{\left\{\left|X_{t}\right| \geq c\right\}}\right] \leq \mathbb{E}\left[\left(2\left|X_{T}\right|-c\right)^{+}\right] .
$$

Now, by dominated convergence,

$$
\lim _{c \rightarrow+\infty} \mathbb{E}\left[\left(2\left|X_{T}\right|-c\right)^{+}\right]=0 .
$$

Hence, property 3 holds.

\subsection{Examples}

The following examples are given in [5] for $d=1$. The proofs given below are essentially the same as in [5].

Proposition 2.3. Let $X$ be a centered $\mathbb{R}^{d}$-valued random variable. Then $(t X, t \geq 0)$ is a peacock.

Proof. Let $\psi: \mathbb{R}^{d} \longrightarrow \mathbb{R}$ be a convex function, and $0 \leq s<t$. Then,

$$
\psi(s X) \leq\left(1-\frac{s}{t}\right) \psi(0)+\frac{s}{t} \psi(t X) .
$$

Since $X$ is centered, by Jensen's inequality:

$$
\psi(0)=\psi(\mathbb{E}[t X]) \leq \mathbb{E}[\psi(t X)] .
$$

Hence,

$$
\mathbb{E}[\psi(s X)] \leq\left(1-\frac{s}{t}\right) \mathbb{E}[\psi(t X)]+\frac{s}{t} \mathbb{E}[\psi(t X)]=\mathbb{E}[\psi(t X)] .
$$

Proposition 2.4. Let $\left(X_{t}, t \geq 0\right)$ be a family of centered, $\mathbb{R}^{d}$-valued, Gaussian variables. We denote by $C(t)=$ $\left(c_{i, j}(t)\right)_{1 \leq i, j \leq d}$ the covariance matrix of $X_{t}$. Then, $\left(X_{t}, t \geq 0\right)$ is a peacock if and only if the map: $t \longrightarrow C(t)$ is increasing in the sense of quadratic forms, i.e.:

$$
\forall a=\left(a_{1}, \ldots, a_{d}\right) \in \mathbb{R}^{d}, \quad t \longrightarrow \sum_{1 \leq i, j \leq d} c_{i, j}(t) a_{i} a_{j} \quad \text { is increasing. }
$$

Proof.

(1) For every $a \in \mathbb{R}^{d}$, the function:

$$
x \in \mathbb{R}^{d} \longrightarrow \sum_{1 \leq i, j \leq d} a_{i} a_{j} x_{i} x_{j}=\left(\sum_{i=1}^{d} a_{i} x_{i}\right)^{2}
$$

is convex. This entails that, if $\left(X_{t}, t \geq 0\right)$ is a peacock, then the map: $t \longrightarrow C(t)$ is increasing in the sense of quadratic forms. 
(2) Conversely, suppose that the map: $t \longrightarrow C(t)$ is increasing in the sense of quadratic forms. By the proof of [5], Theorem 2.16, page 132, there exists a centered $\mathbb{R}^{d}$-valued Gaussian process: $\left(\Gamma_{t}=\left(\Gamma_{1, t}, \ldots, \Gamma_{d, t}\right), t \geq 0\right)$, such that:

$$
\forall s, t \geq 0, \quad \forall 1 \leq i, j \leq d, \quad \mathbb{E}\left[\Gamma_{i, s} \Gamma_{j, t}\right]=c_{i, j}(s \wedge t) .
$$

Therefrom we deduce that $\left(\Gamma_{t}, t \geq 0\right)$ is a martingale which is associated to $\left(X_{t}, t \geq 0\right)$, and consequently, $\left(X_{t}, t \geq 0\right)$ is a peacock.

Corollary 2.5. Let $A$ be a $d \times d$ matrix. We consider the $\mathbb{R}^{d}$-valued Ornstein-Uhlenbeck process $\left(U_{t}, t \geq 0\right)$, defined as (the unique) solution, started from 0 , of the SDE:

$$
\mathrm{d} U_{t}=\mathrm{d} B_{t}+A U_{t} \mathrm{~d} t
$$

where $\left(B_{t}, t \geq 0\right)$ denotes a d-dimensional Brownian motion. Then, $\left(U_{t}, t \geq 0\right)$ is a peacock.

Proof. One has:

$$
U_{t}=\int_{0}^{t} \exp ((t-s) A) \mathrm{d} B_{s} .
$$

Hence, for every $t \geq 0, U_{t}$ is a centered, $\mathbb{R}^{d}$-valued Gaussian variable whose covariance matrix is:

$$
C(t)=\int_{0}^{t} \exp (s A) \exp \left(s A^{*}\right) \mathrm{d} s
$$

where $A^{*}$ denotes the transpose matrix of $A$. Therefrom it is clear that the map: $t \longrightarrow C(t)$ is increasing in the sense of quadratic forms, and Proposition 2.4 applies.

Proposition 2.6. Let $\left(M_{t}, t \geq 0\right)$ be an $\mathbb{R}^{d}$-valued, right-continuous martingale such that:

$$
\forall T>0, \quad \mathbb{E}\left[\sup _{0 \leq t \leq T}\left|M_{t}\right|\right]<\infty
$$

Then,

1. $\left(X_{t}:=\frac{1}{t} \int_{0}^{t} M_{s} \mathrm{~d} s ; t \geq 0\right)$ is a peacock;

2. $\left(\widetilde{X}_{t}:=\int_{0}^{t}\left(M_{s}-M_{0}\right) \mathrm{d} s ; t \geq 0\right)$ is a peacock.

Proof. Using Proposition 2.1, we may use the proof of [5], Theorem 1.4, page 26. For the convenience of the reader, we reproduce this proof below.

(1) Let $\psi: \mathbb{R}^{d} \longrightarrow \mathbb{R}$ be a convex function, of $C^{\infty}$ class and such that the derivative $\psi^{\prime}$ is bounded on $\mathbb{R}^{d}$. Setting:

$$
\widehat{M}_{t}=\int_{0}^{t} s \mathrm{~d} M_{s}
$$

one has, by integration by parts:

$$
X_{t}=M_{t}-t^{-1} \widehat{M}_{t} \quad \text { and } \quad \mathrm{d} X_{t}=t^{-2} \widehat{M}_{t} \mathrm{~d} t .
$$

Denoting by $\mathcal{F}_{s}$ the $\sigma$-algebra generated by $\left\{M_{u} ; 0 \leq u \leq s\right\}$, one gets, for $0 \leq s \leq t$,

$$
\mathbb{E}\left[X_{t} \mid \mathcal{F}_{s}\right]=X_{s}+\left(s^{-1}-t^{-1}\right) \widehat{M}_{s} .
$$


Consequently, by Jensen's inequality,

$$
\mathbb{E}\left[\psi\left(X_{t}\right)\right] \geq \mathbb{E}\left[\psi\left(X_{s}+\left(s^{-1}-t^{-1}\right) \widehat{M}_{s}\right)\right] .
$$

Using again the fact that $\psi$ is convex, one obtains:

$$
\mathbb{E}\left[\psi\left(X_{t}\right)\right] \geq \mathbb{E}\left[\psi\left(X_{s}\right)\right]+\left(s^{-1}-t^{-1}\right) \mathbb{E}\left[\psi^{\prime}\left(X_{s}\right) \cdot \widehat{M}_{s}\right] .
$$

Now,

and therefore

$$
\psi^{\prime}\left(X_{s}\right) \cdot \widehat{M}_{s}=\int_{0}^{s} u^{-2} \psi^{\prime \prime}\left(X_{u}\right)\left(\widehat{M}_{u}, \widehat{M}_{u}\right) \mathrm{d} u+\int_{0}^{s} u \psi^{\prime}\left(X_{u}\right) \cdot \mathrm{d} M_{u}
$$

$$
\mathbb{E}\left[\psi\left(X_{t}\right)\right]-\mathbb{E}\left[\psi\left(X_{s}\right)\right] \geq\left(s^{-1}-t^{-1}\right) \mathbb{E}\left[\psi^{\prime}\left(X_{s}\right) \cdot \widehat{M}_{s}\right] \geq 0,
$$

which, by Proposition 2.1, yields the desired result.

(2) Let $\psi$ be as above. One may suppose that $M_{0}=0$. One has, for $0 \leq s \leq t$,

$$
\mathbb{E}\left[\widetilde{X}_{t} \mid \mathcal{F}_{s}\right]=\widetilde{X}_{s}+(t-s) M_{s}
$$

Consequently, by Jensen's inequality,

$$
\mathbb{E}\left[\psi\left(\widetilde{X}_{t}\right)\right] \geq \mathbb{E}\left[\psi\left(\widetilde{X}_{s}+(t-s) M_{s}\right)\right] .
$$

Using again the fact that $\psi$ is convex, one obtains:

$$
\mathbb{E}\left[\psi\left(\widetilde{X}_{t}\right)\right] \geq \mathbb{E}\left[\psi\left(\widetilde{X}_{s}\right)\right]+(t-s) \mathbb{E}\left[\psi^{\prime}\left(\widetilde{X}_{s}\right) \cdot M_{s}\right] .
$$

Now,

and therefore

$$
\psi^{\prime}\left(\widetilde{X}_{s}\right) \cdot M_{s}=\int_{0}^{s} \psi^{\prime \prime}\left(\widetilde{X}_{u}\right)\left(M_{u}, M_{u}\right) \mathrm{d} u+\int_{0}^{s} \psi^{\prime}\left(\widetilde{X}_{u}\right) \cdot \mathrm{d} M_{u}
$$

$$
\mathbb{E}\left[\psi\left(\widetilde{X}_{t}\right)\right]-\mathbb{E}\left[\psi\left(\widetilde{X}_{s}\right)\right] \geq(t-s) \mathbb{E}\left[\psi^{\prime}\left(\widetilde{X}_{s}\right) \cdot M_{s}\right] \geq 0,
$$

which, by Proposition 2.1, yields the desired result.

\section{Right-COntinuous PEACOKS}

In this section, we shall show that any right continuous peacock admits an associated right-continuous martingale. For this, we start from Strassen's theorem, which we now recall.

Theorem 3.1 (Strassen [8], Thm. 8). Let $\left(\mu_{n}, n \in \mathbb{N}\right)$ be a sequence in $\mathcal{M}$. Then $\left(\mu_{n}, n \in \mathbb{N}\right)$ is a peacock if and only if there exists a martingale $\left(M_{n}, n \in \mathbb{N}\right)$ which is associated to $\left(\mu_{n}, n \in \mathbb{N}\right)$.

We shall extend this theorem to right-continuous peacocks indexed by $\mathbb{R}_{+}$. In the case $d=1$, the following theorem is proven in [4], by a quite different method. In particular, in [4], we do not use Strassen's theorem, nor the Hahn-Banach theorem, but an explicit approximation by solutions of SDE's.

Theorem 3.2. Let $\left(\mu_{t}, t \geq 0\right)$ be a family in $\mathcal{M}$. Then the following properties are equivalent:

(i) there exists a right-continuous martingale associated to $\left(\mu_{t}, t \geq 0\right)$;

(ii) $\left(\mu_{t}, t \geq 0\right)$ is a peacock and the map:

$$
t \geq 0 \longrightarrow \mu_{t} \in \mathcal{M}
$$

is right-continuous. 
Proof.

(1) We first assume that property (i) is satisfied. Then, the fact that $\left(\mu_{t}, t \geq 0\right)$ is a peacock follows classically from Jensen's inequality. Let $\left(M_{t}, t \geq 0\right)$ be a right-continuous martingale associated to $\left(\mu_{t}, t \geq 0\right)$. Then, if $f \in C_{b}\left(\mathbb{R}^{d}\right)$, dominated convergence yields that, for any $t \geq 0$,

$$
\lim _{s \rightarrow t, s>t} \int f(x) \mu_{s}(\mathrm{~d} x)=\lim _{s \rightarrow t, s>t} \mathbb{E}\left[f\left(M_{s}\right)\right]=\mathbb{E}\left[f\left(M_{t}\right)\right]=\int f(x) \mu_{t}(\mathrm{~d} x) .
$$

Therefore, the map:

$$
t \geq 0 \longrightarrow \mu_{t} \in \mathcal{M}
$$

is right-continuous, and property (ii) is satisfied.

(2) Conversely, we now assume that property (ii) is satisfied. For every $n \in \mathbb{N}$, we set:

$$
\mu_{k}^{(n)}=\mu_{k 2^{-n}}, \quad k \in \mathbb{N}
$$

By Strassen's theorem (Thm. 3.1), there exists a martingale $\left(M_{k}^{(n)}, k \in \mathbb{N}\right)$ which is associated to $\left(\mu_{k}^{(n)}, k \in\right.$ $\mathbb{N})$. We set:

$$
X_{t}^{(n)}=M_{k}^{(n)} \text { if } t=k 2^{-n} \text { and } X_{t}^{(n)}=0 \text { otherwise. }
$$

Consequently, the law of $X_{t}^{(n)}$ is $\mu_{t}$ if $t \in\left\{k 2^{-n} ; k \in \mathbb{N}\right\}$, and is $\delta$ (the Dirac measure at 0 ) if $t \notin\left\{k 2^{-n} ; k \in\right.$ $\mathbb{N}\}$.

Note that, due to the lack of uniqueness in Strassen's theorem, the law of $\left(X_{k 2^{-n}}^{(n)}, k \in \mathbb{N}\right)$ may not be the same as the law of $\left(X_{k 2^{-n}}^{(n+1)}, k \in \mathbb{N}\right)$.

Only the one-dimensional marginals are identical.

(3) Let $D=\left\{k 2^{-n} ; k, n \in \mathbb{N}\right\}$ the set of dyadic numbers. For every $n \in \mathbb{N}$, for every $r \geq 1$ and $\tau_{r}=$ $\left(t_{1}, t_{2}, \ldots, t_{r}\right) \in D^{r}$, we denote by $\Pi_{\tau_{r}}^{(r, n)}$ the law of $\left(X_{t_{1}}^{(n)}, \ldots, X_{t_{r}}^{(n)}\right)$, a probability on $\left(\mathbb{R}^{d}\right)^{r}$.

Lemma 3.3. For every $\tau_{r} \in D^{r}$, the set of probability measures: $\left\{\Pi_{\tau_{r}}^{(r, n)} ; n \in \mathbb{N}\right\}$ is tight.

Proof. We set, for $x=\left(x^{1}, \ldots, x^{r}\right) \in\left(\mathbb{R}^{d}\right)^{r},|x|_{r}=\sum_{j=1}^{r}\left|x^{j}\right|$. Then, for $p>0$,

$$
\Pi_{\tau_{r}}^{(r, n)}\left(|x|_{r} \geq p\right) \leq \frac{1}{p} \Pi_{\tau_{r}}^{(r, n)}\left(|x|_{r}\right)=\frac{1}{p} \sum_{j=1}^{r} \mathbb{E}\left[\left|X_{t_{j}}^{(n)}\right|\right] \leq \frac{1}{p} \sum_{j=1}^{r} \mu_{t_{j}}(|x|)
$$

since, by point (2), the law of $X_{t_{j}}^{(n)}$ is either $\mu_{t_{j}}$ or $\delta$. Hence,

$$
\lim _{p \rightarrow \infty} \sup _{n \geq 0} \Pi_{\tau_{r}}^{(r, n)}\left(|x|_{r} \geq p\right)=0
$$

(4) As a consequence of the previous lemma, and with the help of the diagonal procedure, there exists a subsequence $\left(n_{l}\right)_{l \geq 0}$ such that, for every $\tau_{r} \in D^{r}$, the sequence of probabilities on $\left(\mathbb{R}^{d}\right)^{r}:\left(\Pi_{\tau_{r}}^{\left(r, n_{l}\right)}, l \geq 0\right)$, weakly converges to a probability which we denote by $\Pi_{\tau_{r}}^{(r)}$. We remark that, for $l$ large enough, the law of $X_{t_{j}}^{\left(n_{l}\right)}$ is $\mu_{t_{j}}$. Then, there exists an $\mathbb{R}^{d}$-valued process $\left(X_{t}, t \in D\right)$ such that, for every $r \geq 1$ and every $\tau_{r}=\left(t_{1}, \ldots, t_{r}\right) \in D^{r}$, the law of $\left(X_{t_{1}}, \ldots, X_{t_{r}}\right)$ is $\Pi_{\tau_{r}}^{(r)}$, and $\Pi_{t}^{(1)}=\mu_{t}$ for every $t \in D$. 
Lemma 3.4. The process $\left(X_{t}, t \in D\right)$ is a martingale associated to $\left(\mu_{t}, t \in D\right)$.

Proof. As we have already seen, the process $\left(X_{t}, t \in D\right)$ is associated to $\left(\mu_{t}, t \in D\right)$. We now prove that it is a martingale. We set:

$$
\forall p>0, \quad \forall x \in \mathbb{R}^{d}, \quad \varphi_{p}(x)=\left(1 \vee \frac{|x|}{p}\right)^{-1} x .
$$

Then,

$$
\varphi_{p} \in C_{b}\left(\mathbb{R}^{d} ; \mathbb{R}^{d}\right) \quad \text { and } \quad \varphi_{p}(x)=x \text { for }|x| \leq p .
$$

Let $0 \leq s_{1}<s_{2}<\ldots<s_{r} \leq s \leq t$ be elements of $D$, and let $f \in C_{b}\left(\left(\mathbb{R}^{d}\right)^{r}\right)$. We set: $\|f\|_{\infty}=\sup \{|f(x)| ; x \in$ $\left.\left(\mathbb{R}^{d}\right)^{r}\right\}$. Then, for $l$ large enough,

$$
\mathbb{E}\left[f\left(X_{s_{1}}^{\left(n_{l}\right)}, \ldots, X_{s_{r}}^{\left(n_{l}\right)}\right) X_{t}^{\left(n_{l}\right)}\right]=\mathbb{E}\left[f\left(X_{s_{1}}^{\left(n_{l}\right)}, \ldots, X_{s_{r}}^{\left(n_{l}\right)}\right) X_{s}^{\left(n_{l}\right)}\right] .
$$

On the other hand,

$$
\begin{array}{r}
\left|\mathbb{E}\left[f\left(X_{s_{1}}, \ldots, X_{s_{r}}\right) \varphi_{p}\left(X_{t}\right)\right]-\mathbb{E}\left[f\left(X_{s_{1}}, \ldots, X_{s_{r}}\right) X_{t}\right]\right| \leq\|f\|_{\infty} \mu_{t}\left(|x| 1_{\{|x| \geq p\}}\right), \text { for every } p>0, \\
\left|\mathbb{E}\left[f\left(X_{s_{1}}^{\left(n_{l}\right)}, \ldots, X_{s_{r}}^{\left(n_{l}\right)}\right) \varphi_{p}\left(X_{t}^{\left(n_{l}\right)}\right)\right]-\mathbb{E}\left[f\left(X_{s_{1}}^{\left(n_{l}\right)}, \ldots, X_{s_{r}}^{\left(n_{l}\right)}\right) X_{t}^{\left(n_{l}\right)}\right]\right| \leq\|f\|_{\infty} \mu_{t}\left(|x| 1_{\{|x| \geq p\}}\right), \\
\text { for every } l \text { and every } p>0
\end{array}
$$

and likewise, replacing $t$ by $s$. Moreover,

$$
\lim _{l \rightarrow \infty} \mathbb{E}\left[f\left(X_{s_{1}}^{\left(n_{l}\right)}, \ldots, X_{s_{r}}^{\left(n_{l}\right)}\right) \varphi_{p}\left(X_{t}^{\left(n_{l}\right)}\right)\right]=\mathbb{E}\left[f\left(X_{s_{1}}, \ldots, X_{s_{r}}\right) \varphi_{p}\left(X_{t}\right)\right],
$$

and likewise, replacing $t$ by $s$. Finally, we obtain, for $p>0$,

$$
\left|\mathbb{E}\left[f\left(X_{s_{1}}, \ldots, X_{s_{r}}\right) X_{t}\right]-\mathbb{E}\left[f\left(X_{s_{1}}, \ldots, X_{s_{r}}\right) X_{s}\right]\right| \leq 2\|f\|_{\infty}\left[\mu_{t}\left(|x| 1_{\{|x| \geq p\}}\right)+\mu_{s}\left(|x| 1_{\{|x| \geq p\}}\right)\right],
$$

and the desired result follows, letting $p$ go to $\infty$.

(5) By the classical theory of martingales (see, for example, [2]), almost surely, for every $t \geq 0$,

$$
M_{t}=\lim _{s \rightarrow t, s \in D, s>t} X_{s}
$$

is well defined, and $\left(M_{t}, t \geq 0\right)$ is a right-continuous martingale. Besides, since, by hypothesis, the map: $t \geq 0 \longrightarrow \mu_{t} \in \mathcal{M}$ is right-continuous, we deduce from Lemma 3.4 that this martingale $\left(M_{t}, t \geq 0\right)$ is associated to $\left(\mu_{t}, t \geq 0\right)$.

\section{The General CASE}

Theorem 3.2 shall now be extended, by approximation, to the general case.

Theorem 4.1. Let $\left(\mu_{t}, t \geq 0\right)$ be a family in $\mathcal{M}$. Then the following properties are equivalent:

(i) there exists a martingale associated to $\left(\mu_{t}, t \geq 0\right)$;

(ii) $\left(\mu_{t}, t \geq 0\right)$ is a peacock. 
Proof. Let $\left(\mu_{t}, t \geq 0\right)$ be a peacock.

Lemma 4.2. There exists a countable set $\Delta \subset \mathbb{R}_{+}$such that the map:

$$
t \longrightarrow \mu_{t} \in \mathcal{M}
$$

is continuous at any $s \notin \Delta$.

Proof. Let $\chi: \mathbb{R}^{d} \longrightarrow \mathbb{R}_{+}$be defined by:

$$
\chi(x)=(1-|x|)^{+}=(1 \vee|x|)-|x| .
$$

Then $\chi \in C_{c}^{+}\left(\mathbb{R}^{d}\right)$ and $\chi$ is the difference of two convex functions. We set: $\chi_{m}(x)=m^{d} \chi(m x)$, and we define the countable set $\mathcal{H}$ by:

$$
\mathcal{H}=\left\{\sum_{j=0}^{r} a_{j} \chi_{m}\left(x-q_{j}\right) ; r \in \mathbb{N}, m \in \mathbb{N}, a_{j} \in \mathbb{Q}_{+}, q_{j} \in \mathbb{Q}^{d}\right\} .
$$

For $h \in \mathcal{H}$, the function: $t \longrightarrow \mu_{t}(h)$ is the difference of two increasing functions, and hence admits a countable set $\Delta_{h}$ of discontinuities. We set $\Delta=\bigcup_{h \in \mathcal{H}} \Delta_{h}$. Then $\Delta$ is a countable subset of $\mathbb{R}_{+}$, and $t \longrightarrow \mu_{t}(h)$ is continuous at any $s \notin \Delta$, for every $h \in \mathcal{H}$. Now, it is easy to see that $\mathcal{H}$ is dense in $C_{c}^{+}\left(\mathbb{R}^{d}\right)$ in the following sense: for every $\varphi \in C_{c}^{+}\left(\mathbb{R}^{d}\right)$, there exist a compact set $K \subset \mathbb{R}^{d}$ and a sequence $\left(h_{n}\right)_{n \geq 0} \subset \mathcal{H}$ such that:

$$
\forall n, \quad \operatorname{Supp} h_{n} \subset K \text { and } \lim _{n \rightarrow \infty} h_{n}=\varphi \text { uniformly. }
$$

Consequently, $t \longrightarrow \mu_{t}$ is vaguely continuous at any $s \notin \Delta$, and, since measures $\mu_{t}$ are probabilities, $t \longrightarrow \mu_{t}$ is also weakly continuous at any $s \notin \Delta$.

We may write $\Delta=\left\{d_{j} ; j \in \mathbb{N}\right\}$. For $n \in \mathbb{N}$, we denote by $\left(k_{l}^{(n)}, l \geq 0\right)$ the increasing rearrangement of the set:

$$
\left\{k 2^{-n} ; k \in \mathbb{N}\right\} \cup\left\{d_{j} ; 0 \leq j \leq n\right\} .
$$

We define $\left(\mu_{t}^{(n)}, t \geq 0\right)$ by:

$$
\mu_{t}^{(n)}=\frac{k_{l+1}^{(n)}-t}{k_{l+1}^{(n)}-k_{l}^{(n)}} \mu_{k_{l}^{(n)}}+\frac{t-k_{l}^{(n)}}{k_{l+1}^{(n)}-k_{l}^{(n)}} \mu_{k_{l+1}^{(n)}} \quad \text { if } t \in\left[k_{l}^{(n)}, k_{l+1}^{(n)}\right] .
$$

Lemma 4.3. The following properties hold:

1. for every $n \geq 0,\left(\mu_{t}^{(n)}, t \geq 0\right)$ is a peacock and the map: $t \longrightarrow \mu_{t}^{(n)} \in \mathcal{M}$ is continuous;

2. for any $t \geq 0, \sup \left\{\mu_{t}^{(n)}(|x|) ; n \in \mathbb{N}\right\}<\infty$;

3. for any $t \geq 0$, the set $\left\{\mu_{t}^{(n)} ; n \in \mathbb{N}\right\}$ is uniformly integrable;

4. for $t \geq 0, \lim _{n \rightarrow \infty} \mu_{t}^{(n)}=\mu_{t} \quad$ in $\mathcal{M}$.

Proof. Properties 1 and 4 are clear by construction. Property 2 (resp. property 3 ) follows directly from property 2 (resp. property 3) in Proposition 2.2.

By Theorem 3.2, there exists, for each $n$, a right-continuous martingale $\left(M_{t}^{(n)}, t \geq 0\right)$ which is associated to $\left(\mu_{t}^{(n)}, t \geq 0\right)$. For any $r \in \mathbb{N}$ and $\tau_{r}=\left(t_{1}, \ldots, t_{r}\right) \in \mathbb{R}_{+}^{r}$, we denote by $\Pi_{\tau_{r}}^{(r, n)}$ the law of $\left(M_{t_{1}}^{(n)}, \ldots, M_{t_{r}}^{(n)}\right)$, a probability measure on $\left(\mathbb{R}^{d}\right)^{r}$. 
Lemma 4.4. For every $\tau_{r} \in \mathbb{R}_{+}^{r}$, the set of probability measures: $\left\{\Pi_{\tau_{r}}^{(r, n)} ; n \in \mathbb{N}\right\}$ is tight.

Proof. As in Lemma 3.3, for $p>0$,

$$
\Pi_{\tau_{r}}^{(r, n)}\left(|x|_{r} \geq p\right) \leq \frac{1}{p} \sum_{j=1}^{r} \mu_{t_{j}}^{(n)}(|x|),
$$

and by property 2 in Lemma 4.3 ,

$$
\lim _{p \rightarrow \infty} \sup _{n \geq 0} \Pi_{\tau_{r}}^{(r, n)}\left(|x|_{r} \geq p\right)=0 .
$$

Let now $\mathcal{U}$ be an ultrafilter on $\mathbb{N}$, which refines Fréchet's filter. As a consequence of the previous lemma, for every $r \in \mathbb{N}$ and every $\tau_{r} \in \mathbb{R}_{+}^{r}, \lim _{\mathcal{U}} \Pi_{\tau_{r}}^{(r, n)}$ exists for the weak convergence and we denote this limit by $\Pi_{\tau_{r}}^{(r)}$. By property 4 in Lemma $4.3, \Pi_{t}^{(1)}=\mu_{t}$. There exists a process $\left(M_{t}, t \geq 0\right)$ such that, for every $r \in \mathbb{N}$ and every $\tau_{r}=\left(t_{1}, \ldots, t_{r}\right) \in \mathbb{R}_{+}^{r}$, the law of $\left(M_{t_{1}}, \ldots, M_{t_{r}}\right)$ is $\Pi_{\tau_{r}}^{(r)}$. In particular, this process $\left(M_{t}, t \geq 0\right)$ is associated to $\left(\mu_{t}, t \geq 0\right)$.

Lemma 4.5. The process $\left(M_{t}, t \geq 0\right)$ is a martingale.

Proof. The proof is quite similar to that of Lemma 3.4, but we give the details for the sake of completeness. We recall the notation:

$$
\forall p>0, \quad \forall x \in \mathbb{R}^{d}, \quad \varphi_{p}(x)=\left(1 \vee \frac{|x|}{p}\right)^{-1} x .
$$

Let $0 \leq s_{1}<s_{2}<\ldots<s_{r} \leq s \leq t$ be elements of $\mathbb{R}_{+}$, and let $f \in C_{b}\left(\left(\mathbb{R}^{d}\right)^{r}\right)$. We set: $\|f\|_{\infty}=\sup \{|f(x)| ; x \in$ $\left.\left(\mathbb{R}^{d}\right)^{r}\right\}$. Then, for every $n$,

$$
\mathbb{E}\left[f\left(M_{s_{1}}^{(n)}, \ldots, M_{s_{r}}^{(n)}\right) M_{t}^{(n)}\right]=\mathbb{E}\left[f\left(M_{s_{1}}^{(n)}, \ldots, M_{s_{r}}^{(n)}\right) M_{s}^{(n)}\right] .
$$

On the other hand,

$$
\begin{array}{r}
\left|\mathbb{E}\left[f\left(M_{s_{1}}, \ldots, M_{s_{r}}\right) \varphi_{p}\left(M_{t}\right)\right]-\mathbb{E}\left[f\left(M_{s_{1}}, \ldots, M_{s_{r}}\right) M_{t}\right]\right| \leq\|f\|_{\infty} \mu_{t}\left(|x| 1_{\{|x| \geq p\}}\right), \\
\left|\mathbb{E}\left[f\left(M_{s_{1}}^{(n)}, \ldots, M_{s_{r}}^{(n)}\right) \varphi_{p}\left(M_{t}^{(n)}\right)\right]-\mathbb{E}\left[f\left(M_{s_{1}}^{(n)}, \ldots, M_{s_{r}}^{(n)}\right) M_{t}^{(n)}\right]\right| \leq\|f\|_{\infty} \mu_{t}^{(n)}\left(|x| 1_{\{|x| \geq p\}}\right) \\
\text { for every } n \text { and every } p>0
\end{array}
$$

and likewise, replacing $t$ by $s$. Moreover,

$$
\lim _{\mathcal{U}} \mathbb{E}\left[f\left(M_{s_{1}}^{(n)}, \ldots, M_{s_{r}}^{(n)}\right) \varphi_{p}\left(M_{t}^{(n)}\right)\right]=\mathbb{E}\left[f\left(M_{s_{1}}, \ldots, M_{s_{r}}\right) \varphi_{p}\left(M_{t}\right)\right]
$$

and likewise, replacing $t$ by $s$. Finally, we obtain, for $p>0$,

$$
\left|\mathbb{E}\left[f\left(X_{s_{1}}, \ldots, X_{s_{r}}\right) X_{t}\right]-\mathbb{E}\left[f\left(X_{s_{1}}, \ldots, X_{s_{r}}\right) X_{s}\right]\right| \leq 2\|f\|_{\infty} \sup _{n \geq 0}\left[\mu_{t}^{(n)}\left(|x| 1_{\{|x| \geq p\}}\right)+\mu_{s}^{(n)}\left(|x| 1_{\{|x| \geq p\}}\right)\right],
$$

and, by property 3 in Lemma 4.3, the desired result follows, letting $p$ go to $\infty$.

This lemma completes the proof of Theorem 4.1.

Acknowledgements. We are grateful to Marc Yor for his help during the preparation of this paper. 


\section{REFERENCES}

[1] P. Cartier, J.M.G. Fell and P.-A. Meyer, Comparaison des mesures portées par un convexe compact. Bull. Soc. Math. France 92 (1964) 435-445.

[2] C. Dellacherie and P.-A. Meyer, Probabilités et potentiel, in Théorie des martingales, Chapitres V à VIII. Hermann (1980).

[3] J.L. Doob, Generalized sweeping-out and probability. J. Funct. Anal. 2 (1968) 207-225.

[4] F. Hirsch and B. Roynette, A new proof of Kellerer's theorem. ESAIM: PS 16 (2012) 48-60.

[5] F. Hirsch, C. Profeta, B. Roynette and M. Yor, Peacocks and associated martingales, with explicit constructions. Bocconi \& Springer Series 3 (2011).

[6] H.G. Kellerer, Markov-Komposition und eine Anwendung auf Martingale. Math. Ann. 198 (1972) 99-122.

[7] G. Lowther, Fitting martingales to given marginals. http://arxiv.org/abs/0808.2319v1 (2008).

[8] V. Strassen, The existence of probability measures with given marginals. Ann. Math. Stat. 36 (1965) $423-439$. 